\title{
El derecho de la naturaleza de disponer de sus residuos salinos
}

\section{The right of nature to dispose of its salts}

\author{
Víctor M. Ponce ${ }^{1}$ \\ Fernando Oñate-Valdivieso² \\ Raúl Cobos-Aguilar ${ }^{3}$
}

\begin{abstract}
${ }^{1}$ Departamento de Ingeniería Civil, Construcción, y Ambiental; Universidad Estatal de San Diego, California, Estados Unidos, ponce@pon.sdsu.edu

2Departamento de Geología, Minas e Ingeniería Civil; Grupo de Hidrología y Climatología; Universidad Técnica Particular de Loja, Loja, Ecuador, fronate@utpl.edu.ec
\end{abstract}

${ }^{3}$ Facultad de Ingeniería "Arturo Narro Siller", Universidad Autónoma de Tamaulipas, Tampico, Tamaulipas, México,rckobos@gmail.com

Autor para correspondencia: Fernando Rodrigo Oñate Valdivieso, fronate@utpl.edu.ec

\section{Resumen}

Iones de sodio y calcio se producen de forma constante por la desintegración de la litósfera en cantidades muy por encima de las que podrían ser asimiladas por la biósfera terrestre. En drenajes exorreicos, los residuos salinos resultantes han sido (y están siendo) transportados al océano por los ríos. Por otro lado, en drenajes endorreicos, la producción de sal se ha acumulado a través de milenios en el interior de los continentes. En general, el exorreísmo es preferible al endorreísmo, pues lleva a ecosistemas más diversos. La irrigación convierte la escorrentía en evapotranspiración, reduciendo así la cantidad de agua disponible para el lavado de las sales. Al mismo tiempo, en regiones áridas, la irrigación moviliza nuevas sales debido 
al intemperismo adicional en suelos jóvenes. En el límite, cuando toda la escorrentía es secuestrada y convertida en evaporación, no queda ningún agua para llevar las sales al océano. De manera eventual, el sistema se vuelve insostenible, no por falta de agua para la vegetación, sino por falta de agua para la eliminación de las sales. Siguiendo los principios de hidrología, aquí postulamos que la naturaleza tenía un plan diferente: en promedio, dos tercios del agua disponible para el ecosistema, y el tercio restante para el lavado de las sales. La estrategia equivocada de retener toda la escorrentía con el argumento de que constituye un recurso "muy valioso" sólo puede conducir a la eventual conversión de las cuencas continentales periféricas en cuencas artificialmente infestadas con sal.

Palabras clave: sal, salinización, derecho de la naturaleza, balance de sales.

\section{Abstract}

Ions of sodium and calcium are produced by lithospheric disintegration in quantities far exceeding those that could possibly be assimilated by the terrestrial biosphere. In exorheic drainages, the resulting waste has been (and is being) transported to the ocean by streams and rivers. In endorheic drainages, it has collected in the interior of continents, producing closed salt basins. In general, exorheism is preferable to endorheism because it leads to healthier and more diverse ecosystems. Irrigation converts runoff into evaporation, reducing the amount of water available for salt flushing. At the same time, particularly in arid and semiarid regions where it is most needed, irrigation mobilizes new salts through the additional biodegradation of geologically young soils. At the limit, when all runoff is sequestered and converted to evaporation, there is no water left to carry the salts to the ocean. As the system is gradually driven towards this limit, it becomes unsustainable, not for lack of water to support the vegetation, but for lack of water to flush the salts. In accordance with hydrologic science, we posit that nature had a different plan: on average, two-thirds of the available water for the ecosystem and the remaining one-third for flushing all salts, regional and new. The misguided strategy of retaining all runoff, on the grounds that it constitutes "precious" water, could only lead to the eventual conversion of peripheral continental basins into artificial, salt-infested basins.

Keywords: Salt, salinization, right of nature, salt balance.

Recibido: 24/12/2015 
Aceptado: 02/11/2017

\section{El origen de las sales del océano}

A nivel global, la cantidad total de agua se ha estimado en 1500 millones de $\mathrm{km}^{3}$ (Penman, 1970). De este total, un 97\%, o 1455 millones, corresponde a los océanos. Los océanos contienen sales disueltas en concentraciones medias de $35 \mathrm{~kg} / \mathrm{m}^{3}$. Por lo tanto, la cantidad total de sal en los océanos es de unas $50 \times 10^{15}$ toneladas métricas, lo cual representa una cantidad enorme (Swenson, 2015). No se conoce a ciencia cierta cuál es el origen de toda esta sal; sin embargo, la mayor parte de ella debe haberse originado en las rocas terrestres (es decir, en la litósfera), intemperizada en el tiempo geológico, y transportada a los océanos por el flujo incesante de los ríos (Pillsbury, 1981).

De todos los iones de sal disueltos en los océanos, el sodio $(\mathrm{Na}+)$ y el cloro ( $\mathrm{Cl}-$ ) son la mayoría, constituyendo entre ellos alrededor de cinco sextas $(5 / 6)$ partes, por peso. En forma elemental, tanto el metal de sodio $(\mathrm{Na})$ como el gas de cloro $(\mathrm{Cl})$ son altamente reactivos; sin embargo, cuando se combinan en el cloruro de sodio $(\mathrm{Na} \mathrm{Cl})$, este último resulta siendo altamente estable.

El principal uso industrial del cloruro de sodio es para posibilitar el transporte y comercialización de sus dos componentes. Grandes cantidades de cloruro de sodio son extraídas de las aguas del océano y regiones endorreicas para su uso como materia prima en la producción de cloro $(\mathrm{Cl} 2)$ y soda cáustica $(\mathrm{NaOH})$. Por ejemplo, la Figura 1 muestra la salina de Guerrero Negro, en Baja California Sur, México, considerada como la más grande del mundo. 

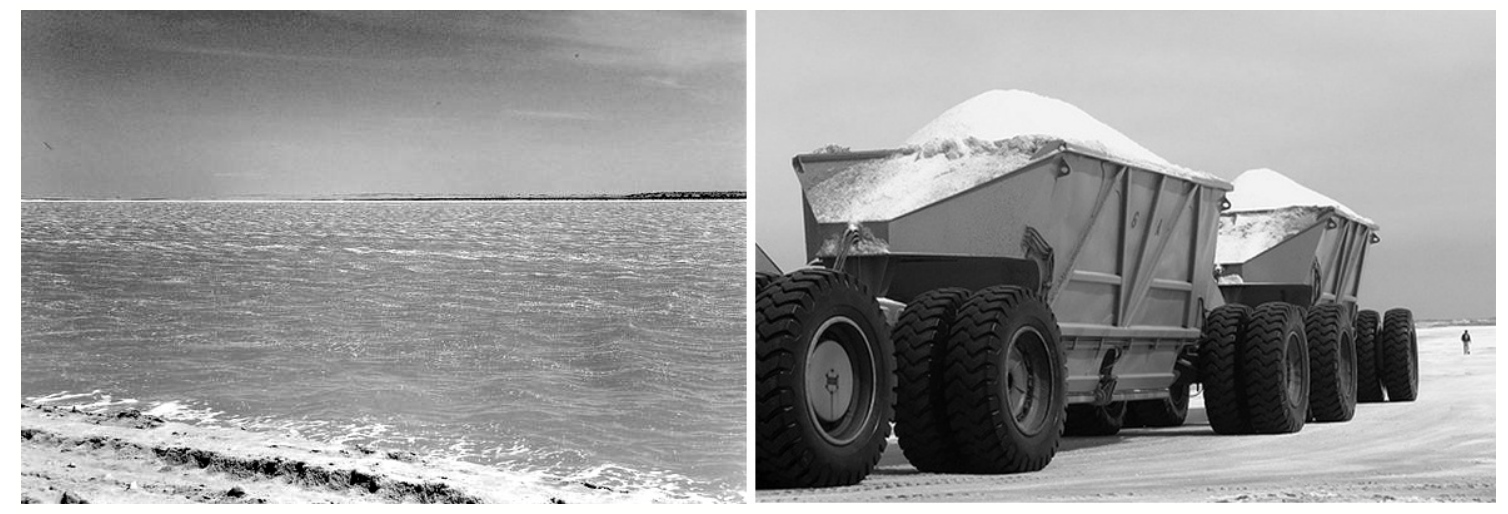

Figura 1. a) Lagunas costeras de evaporación en Guerrero Negro (Visualab, 2015); b) producción de cloruro de sodio a granel en la planta de sal de Guerrero Negro (trotamexico.com, 2015).

\section{Naturaleza de las sales}

Las sales son compuestos químicos que se originan en la reacción de un ácido y una base. Las sales están compuestas de cationes (con carga positiva) y aniones (con carga negativa), de tal manera que la combinación es eléctricamente neutra.

Los iones de sal pueden ser inorgánicos u orgánicos. Los cationes de sales inorgánicas más comunes son el sodio $(\mathrm{Na}+)$, magnesio $(\mathrm{Mg}+)$, potasio $(\mathrm{K}+)$ y calcio $(\mathrm{Ca}+)$. Estos iones se agrupan por número atómico en la Tabla Periódica de los Elementos. Sus números atómicos son $11,12,19$ y 20 , respectivamente.

El anión más común de sal inorgánica es el cloro $(\mathrm{Cl}-)$, con número atómico 17; el otro anión es el fluoruro (F-), con número atómico 9 . El sodio y el cloro son los iones de sal más abundantes en los océanos (Deevey, 1970). En su estado mineral, el cloruro de sodio se conoce como halita; en el uso común se la refiere como sal de mesa.

\section{El origen de las sales}


La naturaleza se compone de cuatro partes: (1) biósfera, (2) litósfera, (3) hidrósfera, y (4) atmósfera. Éstas interaccionan entre sí para formar la ecosfera, la suma total de la naturaleza, abarcando todos sus componentes. La litósfera contiene grandes cantidades de los cuatro cationes de sal más comunes ( $\mathrm{Na}+, \mathrm{Ca}+, \mathrm{Mg}+\mathrm{y} \mathrm{K}+)$; sin embargo, tiene sólo cantidades muy pequeñas de aniones de sal (ion cloro $\mathrm{Cl}-\mathrm{e}$ ion flúor F-) (Deevey, 1970). Por lo tanto, para que se formen los compuestos de sal, los aniones deben ser suministrados por las otras esferas. El ion cloro es suministrado con creces por la hidrósfera (Deevey, 1970). El ion cloro también es proporcionado en pequeñas cantidades (aerosoles) por los océanos a la atmósfera, y con el tiempo éstos regresan a la hidrósfera a través de la precipitación.

La Tabla 1 muestra la presencia relativa de iones en la litósfera. Los iones de mayor concentración en la litósfera son el oxígeno (O-) y el silicio ( $\mathrm{Si}+)$, con 60.4 y $20.5 \%$ de átomos, respectivamente. Los cationes de sales inorgánicas $(\mathrm{Na}+, \mathrm{Ca}+, \mathrm{Mg}+, \mathrm{K}+)$ constituyen el $2.49,1.88,1.77$, y $1.37 \%$, de los átomos, respectivamente (Tabla 1 , columna 3) (Deevey, 1970). La columna 7 muestra que los cationes de sales inorgánicas constituyen un $11.1 \%$ en peso de la litósfera. Esto significa que por cada tonelada métrica $(1000 \mathrm{~kg}$ ) de rocas litosféricas desagregadas por meteorización, erosión y otros procesos naturales, unos $111 \mathrm{~kg}$ de cationes de sal son liberados a las otras esferas. La columna 7 muestra que los cuatro cationes de sales inorgánicas están más o menos presentes de manera uniforme en la roca madre.

Tabla 1. Presencia relativa de iones en la litósfera.

\begin{tabular}{|c|c|c|c|c|c|c|}
\hline $\begin{array}{c}\text { Catión o } \\
\text { anión } \\
\text { elemental }\end{array}$ & Símbolo & $\begin{array}{c}\text { \% de } \\
\text { átomo } \\
\mathbf{S}\end{array}$ & $\begin{array}{c}\text { Peso } \\
\text { molecular } \\
\text { (PM) }\end{array}$ & $\begin{array}{c}\% \times \text { PM } \\
\text { (todos } \\
\text { los } \\
\text { cationes) }\end{array}$ & $\begin{array}{c}\% \times \text { PM } \\
\text { (catione } \\
\text { s } \\
\text { de sal) }\end{array}$ & $\begin{array}{c}\text { \% del } \\
\text { total } \\
\text { (sólo } \\
\text { cationes } \\
\text { de sal) }\end{array}$ \\
\hline Oxígeno & $\mathrm{O}^{-}$ & 60.40 & 15.99 & 966.340 & - & - \\
\hline Silicio & $\mathrm{Si}^{+}$ & 20.50 & 28.09 & 575.845 & - & - \\
\hline Aluminio & $\mathrm{Al}^{+}$ & 6.20 & 26.98 & 167.276 & - & - \\
\hline Hidrógeno & $\mathrm{H}^{+}$ & 2.92 & 1.01 & 2.949 & - & - \\
\hline Sodio & $\mathrm{Na}^{+}$ & 2.49 & 22.99 & 57.245 & 57.245 & 2.77 \\
\hline
\end{tabular}




\begin{tabular}{|c|c|c|c|c|c|c|}
\hline Calcio & $\mathrm{Ca}^{+}$ & 1.88 & 40.08 & 75.350 & 75.350 & 3.65 \\
\hline Magnesio & $\mathrm{Mg}^{+}$ & 1.77 & 24.31 & 43.029 & 43.029 & 2.08 \\
\hline Potasio & $\mathrm{K}^{+}$ & 1.37 & 39.10 & 53.567 & 53.567 & 2.59 \\
\hline Hierro & $\mathrm{Fe}^{+}$ & 1.90 & 55.85 & 106.115 & - & - \\
\hline Titanio & $\mathrm{Ti}^{+}$ & 0.27 & 47.90 & 12.933 & - & - \\
\hline Carbono & $\mathrm{C}^{+}$ & 0.16 & 12.01 & 1.922 & - & - \\
\hline Fósforo & $\mathrm{P}^{+}$ & 0.08 & 30.97 & 2.478 & - & - \\
\hline Azufre & $\mathrm{S}^{+}$ & 0.04 & 32.06 & 1.282 & - & - \\
\hline Suma & - & 99.98 & - & 2066.331 & 229.191 & 11.09 \\
\hline
\end{tabular}

¿Qué fracción de esta cantidad (111 kg por tonelada métrica) es incorporado por las otras tres esferas? El sodio está mayormente ausente de la biósfera y atmósfera, pero presente de manera significativa en la hidrósfera. El calcio, magnesio y potasio se encuentran en la biósfera y la hidrósfera, pero ausentes de la atmósfera (nótese que el nitrógeno, un componente muy importante de la biósfera, está en gran medida ausente de la litósfera, siendo la atmósfera su fuente principal) (Deevey, 1970).

Después de su liberación de la litósfera, el sodio es absorbido sólo por la hidrósfera, mientras que calcio, magnesio y potasio son absorbidos tanto por la biósfera como por la hidrósfera. Aparentemente, el sodio no es utilizado por la biósfera en cantidad apreciables, sobre todo en comparación con los otros tres cationes de sal (Deevey, 1970). La Tabla 2 resume estas observaciones.

Tabla 2. Origen y destino de los cuatro cationes más comunes de la sal.

\begin{tabular}{|c|c|c|c|c|c|}
\hline $\begin{array}{c}\text { Catión } \\
\text { elemen- } \\
\text { tal de } \\
\text { sal }\end{array}$ & \multirow{2}{*}{ Símbolo } & Origen & \multicolumn{3}{|c|}{ Destino } \\
\cline { 4 - 6 } & & Biósfera & Hidrósfera & $\begin{array}{c}\text { Atmós- } \\
\text { fera }\end{array}$ \\
\hline Sodio & $\mathrm{Na}^{+}$ & Litósfera & & $\checkmark$ & - \\
\hline Calcio & $\mathrm{Ca}^{+}$ & Litósfera & $\checkmark$ & $\checkmark$ & - \\
\hline Magnesio & $\mathrm{Mg}^{+}$ & Litósfera & $\checkmark$ & $\checkmark$ & - \\
\hline
\end{tabular}




\begin{tabular}{|l|l|l|l|l|l|}
\hline Potasio & $\mathrm{K}^{+}$ & Litósfera & $\checkmark$ & $\checkmark$ & - \\
\hline
\end{tabular}

La evidencia existente sugiere que grandes cantidades de potasio y magnesio son absorbidas por la biósfera terrestre, mientras que la mayoría del calcio y sodio son rechazados por la biósfera, uniéndose a la hidrósfera continental. El potasio es esencial para el funcionamiento de todas las células vivas; asimismo, cabe notar que un átomo de magnesio se encuentra en el centro de cada molécula de clorofila (Deevey, 1970).

La hidrósfera es selectiva en su uso de cationes de sal. La Tabla 3 compara los porcentajes de contenido de sal iónica en el agua de mar, o salada, y en el agua dulce o de río (Swenson, 2015). En el agua de mar, los iones de sal predominantes son cloro $(\mathrm{Cl}-)$ y sodio $(\mathrm{Na}+)$. En el agua dulce, los iones predominantes son bicarbonato (HCO3-), calcio $(\mathrm{Ca}+)$ y sulfato $(\mathrm{SO} 4-)$. Cloro $(\mathrm{Cl}-)$ y sodio $(\mathrm{Na}+)$ son los siguientes en la lista, también con porcentajes considerables.

Hay varias razones para esta selectividad. El bicarbonato y el sulfato aparecen en el agua dulce como los principales productos de la biodegradación. Calcio y sodio no son utilizados de manera significativa por la biósfera, la cual desperdicia el excedente de calcio y de sodio por lixiviación hacia las corrientes de agua dulce. Potasio y magnesio también aparecen en el agua dulce, pero en porcentajes más pequeños que el calcio y sodio.

Tabla 3. Porcentajes de contenido iónico de sales en el agua de mar y agua dulce.

\begin{tabular}{|c|c|c|c|}
\hline Ion & Símbolo & Agua de mar & Agua dulce \\
\hline Cloro & $\mathrm{Cl}^{-}$ & 55.16 & 8.64 \\
\hline Sodio & $\mathrm{Na}^{+}$ & 30.53 & 6.98 \\
\hline Sulfato & $\mathrm{SO}_{4}^{-}$ & 7.67 & 12.41 \\
\hline Magnesio & $\mathrm{Mg}^{+}$ & 3.72 & 4.54 \\
\hline Calcio & $\mathrm{Ca}^{+}$ & 1.19 & 16.62 \\
\hline Potasio & $\mathrm{K}^{+}$ & 1.11 & 2.55 \\
\hline Bicarbonato & $\mathrm{HCO}_{3}^{-}$ & 0.42 & 31.90 \\
\hline
\end{tabular}




\begin{tabular}{|c|c|c|c|}
\hline Bromuro & $\mathrm{Br}^{-}$ & 0.20 & - \\
\hline Silicio & $\mathrm{SiO}_{2}{ }^{-}$ & - & 14.51 \\
\hline Nitrato & $\mathrm{NO}_{3}{ }^{-}$ & - & 1.11 \\
\hline Hierro & $\mathrm{Fe}^{+}$ & - & 0.74 \\
\hline Total & - & $\mathbf{1 0 0 . 0 0}$ & $\mathbf{1 0 0 . 0 0}$ \\
\hline
\end{tabular}

Las Tablas 4a a 4c muestran el porcentaje de los cuatro cationes de sal más comunes en: (a) la litósfera, (b) el agua dulce, y (c) el agua de mar, respectivamente. Nótese que la presencia de potasio y magnesio en la litósfera es mucho mayor que su presencia en el agua dulce. El calcio parece ser poco afectado por la biósfera y lo mismo puede decirse del sodio. Como resultado de la captura de potasio y magnesio por la biósfera, la cantidad de cationes de estas sales inorgánicas efectivamente entregado a los cuerpos de agua dulce es mucho menor que en su fuente litosférica.

Podría considerarse un valor de 50\% como una estimación aproximada del porcentaje de cationes de sal litosférica entregado a los cuerpos de agua dulce. Esto significaría que por cada tonelada métrica de roca intemperizada y erosionada, alrededor de $55 \mathrm{~kg}$ de cationes de sal son entregados al agua dulce. Como comparación, la combustión de una tonelada métrica de materia orgánica produce sólo alrededor de $12 \mathrm{~kg}$ de ceniza (Deevey, 1970). Esto pone de relieve la diferente naturaleza química de la hidrósfera continental (los cuerpos de agua superficial) y la biósfera.

Tabla 4a. Porcentaje de los cuatro cationes de sal más comunes en la litósfera.

\begin{tabular}{|c|c|c|c|c|c|}
\hline $\begin{array}{c}\text { Catión } \\
\text { elemental } \\
\text { de sal }\end{array}$ & Símbolo & $\begin{array}{c}\text { \% de } \\
\text { átomos }\end{array}$ & $\begin{array}{c}\text { Peso } \\
\text { molecu- } \\
\text { lar (PM) }\end{array}$ & $\begin{array}{c}\text { \% de } \\
\text { átomos } \\
\times \text { PM }\end{array}$ & $\begin{array}{c}\text { \% de } \\
\text { cationes de } \\
\text { sal en la } \\
\text { litósfera }\end{array}$ \\
\hline Sodio & $\mathrm{Na}^{+}$ & 2.49 & 22.99 & 57.245 & 24.98 \\
\hline Calcio & $\mathrm{Ca}^{+}$ & 1.88 & 40.08 & 75.350 & 32.88 \\
\hline Magnesio & $\mathrm{Mg}^{+}$ & 1.77 & 24.31 & 43.029 & 18.77 \\
\hline Potasio & $\mathrm{K}^{+}$ & 1.37 & 39.10 & 53.567 & 23.37 \\
\hline Suma & & & & $\mathbf{2 2 9 . 1 9 1}$ & $\mathbf{1 0 0 . 0 0}$ \\
\hline
\end{tabular}


Tabla 4b. Porcentaje de los cuatro cationes de sal más comunes en los cuerpos de agua dulce.

\begin{tabular}{|c|c|c|c|}
\hline $\begin{array}{c}\text { Catión } \\
\text { elemental } \\
\text { de sal }\end{array}$ & Símbolo & $\begin{array}{c}\text { \% de sólidos en } \\
\text { agua dulce }\end{array}$ & $\begin{array}{c}\text { \% de cationes de sal } \\
\text { en agua dulce }\end{array}$ \\
\hline Sodio & $\mathrm{Na}^{+}$ & 6.98 & 22.74 \\
\hline Calcio & $\mathrm{Ca}^{+}$ & 16.62 & 54.16 \\
\hline Magnesio & $\mathrm{Mg}^{+}$ & 4.54 & 14.79 \\
\hline Potasio & $\mathrm{K}^{+}$ & 2.55 & 8.31 \\
\hline Suma & & 30.69 & 100.00 \\
\hline
\end{tabular}

Tabla 4c. Porcentaje de los cuatro cationes de sal más comunes en el agua de mar.

\begin{tabular}{|c|c|c|c|}
\hline $\begin{array}{c}\text { Catión } \\
\text { elemental } \\
\text { de sal }\end{array}$ & Símbolo & $\begin{array}{c}\text { \% de sólidos en } \\
\text { el agua de mar }\end{array}$ & $\begin{array}{c}\text { \% de cationes de sal } \\
\text { en el agua de mar }\end{array}$ \\
\hline Sodio & $\mathrm{Na}^{+}$ & 30.53 & 83.53 \\
\hline Calcio & $\mathrm{Ca}^{+}$ & 1.19 & 3.25 \\
\hline Magnesio & $\mathrm{Mg}^{+}$ & 3.72 & 10.18 \\
\hline Potasio & $\mathrm{K}^{+}$ & 1.11 & 3.04 \\
\hline Suma & & 36.55 & 100.00 \\
\hline
\end{tabular}

Mientras que tanto el calcio como el sodio son desperdiciados por la biósfera terrestre, lo que justifica su presencia en los cuerpos de agua dulce, la ausencia de calcio disuelto en el agua de mar requiere de una justificación adicional (ver Tabla 4c). La contradicción se resuelve cuando se toma en cuenta que la biósfera oceánica es en realidad un sumidero de calcio, empleado por una variedad de organismos marinos para la construcción de conchas y esqueletos (Swenson, 2015).

La Tabla 5 resume los resultados de la Tabla $4(a, b, c)$. El sodio es el único catión inorgánico que permanece en solución en los océanos. No 
existe un proceso biológico conocido que extraiga y use los iones de sodio de los océanos (Swenson, 2015). Junto con el ion cloro, un constituyente de la hidrósfera, estos dos iones representan el $85.7 \%$ del total de sólidos disueltos en el agua de mar (la suma de las dos primeras líneas de la Columna 3, Tabla 3). La extremadamente alta solubilidad del cloruro de sodio en el agua, la más alta de todos los componentes químicos conocidos, garantiza que una vez disuelto en agua corriente permanezca en solución hasta alcanzar su término en los océanos.

Tabla 5. Presencia relativa de los cuatro cationes de sal más comunes en la litósfera e hidrósfera.

\begin{tabular}{|c|c|c|c|c|}
\hline \multirow{2}{*}{$\begin{array}{c}\text { Catión } \\
\text { elemental } \\
\text { de sal }\end{array}$} & \multirow{2}{*}{ Símbolo } & \multicolumn{3}{|c|}{ Porcentaje de presencia en la } \\
\cline { 3 - 5 } & & Litósfera & \multicolumn{2}{c|}{ Hidrósfera } \\
\cline { 3 - 5 } & & 24.98 & 24.74 & 83.53 \\
\hline Sodio & $\mathrm{Na}^{+}$ & 32.88 & 54.16 & 3.25 \\
\hline Calcio & $\mathrm{Ca}^{+}$ & 18.77 & 14.79 & 10.18 \\
\hline Magnesio & $\mathrm{Mg}^{+}$ & 23.37 & 8.31 & 3.04 \\
\hline Potasio & $\mathrm{K}^{+}$ & $\mathbf{1 0 0 . 0 0}$ & $\mathbf{1 0 0 . 0 0}$ & $\mathbf{1 0 0 . 0 0}$ \\
\hline Total & - & & & \\
\hline
\end{tabular}

\section{El derecho de la naturaleza}

Una vez establecido el origen de los iones de sal y aclarado su eventual destino, se concluye que la naturaleza (en este caso, la biósfera terrestre) se ha reservado para sí el derecho de disponer de sus residuos de sal. Los cationes de calcio y sodio son desechados en gran medida por la biósfera terrestre debido a que, al igual que cualquier otro residuo, la oferta es mucho mayor que la demanda. 
Después de llegar al océano, el calcio es utilizado por los organismos marinos, dejando al sodio $-\mathrm{y}$ el cloro, su socio ubicuo- como los únicos verdaderos residuos de la naturaleza. En drenajes continentales periféricos, en los cuales prevalecen condiciones exorreicas, las corrientes de agua dulce son los agentes responsables del lavado de las sales excedentes al océano. Este derecho fundamental de la naturaleza ha sido ejercido desde un principio (Pillsbury, 1981).

En general, las cuencas exorreicas, normalmente ubicadas en la periferia de los continentes, son capaces de entregar sus sales al océano. Contrario a esto, los drenajes endorreicos, por lo usual situados en el interior, son incapaces de eliminar sus sales, las cuales se acumulan en el tiempo geológico. El hecho de que una cuenca sea exorreica o endorreica dependerá de su ubicación continental y de la geomorfología local y regional (Ponce, 2014). Las cuencas o drenajes totalmente exorreicos están en equilibrio de sales, sin acumulación neta de éstas, mientras que los drenajes endorreicos no lo están.

El endorreísmo acumula sales, desalentando el desarrollo de ecosistemas diversos y, por tanto, limitando el desarrollo normal de la vida. Por el contrario, los drenajes exorreicos eliminan las sales excedentes, lo que les permite seguir apoyando a la vida en sus variadas manifestaciones. Cuanto más grande es la masa continental, mayor es la probabilidad de endorreísmo (Figura 2). Los drenajes exorreicos llevan una ventaja sobre los endorreicos con respecto al balance de sales.




Figura 2. El lago Garabogazköl, en Turkmenistan, Asia, el término del drenaje del lago Caspio. La salinidad del lago es de $345 \mathrm{~kg} / \mathrm{m}^{3}$, una de la más altas, y cerca de 10 veces mayor que la salinidad del océano (Google Earth, 2015).

En resumen, en cuencas ubicadas en la periferia de los continentes, los ríos sirven para conducir las sales residuales al océano, donde han de acumularse y permanecer fuera de la vista por todos los tiempos. Entonces, es correcto afirmar que los ríos son exportadores naturales de sal al océano (Pillsbury, 1981). Por lo tanto, la reducción del caudal de los ríos mediante la captación antropogénica, y su eventual conversión en evapotranspiración a través del riego, tiene el efecto de convertir un drenaje exorreico en uno endorreico, comprometiendo así el balance natural de sales. Cabe notar que esto es precisamente lo que los seres humanos han venido haciendo en los últimos tiempos, en particular durante el siglo pasado.

\section{El diseño de la naturaleza}

El ciclo hidrológico es el motor primordial de la naturaleza. El ciclo tiene tres componentes: (1) precipitación, (2) evaporación y (3) escurrimiento. Una vez llegada a la superficie del suelo, la precipitación se separa en escurrimiento y evaporación. A través del escurrimiento, el agua regresa directamente al océano, donde se originó; a través de la evaporación, el agua regresa a la atmósfera, acortando así el ciclo hidrológico.

En promedio global anual, alrededor de una tercera parte $(1 / 3)$ de la precipitación es convertida en escurrimiento, mientras que la fracción restante (2/3) es convertida en vapor de agua y regresada directamente a la atmósfera. Sobre una base anual, la relación de (volumen de) escurrimiento a (volumen de) precipitación se conoce como el coeficiente de escurrimiento, rendimiento anual, o módulo anual de la cuenca $K$. Este coeficiente varía desde valores bajos, $K=$ 0.02 , hasta valores altos, $K=0.93$, esto último en casos muy inusuales (L'vovich, 1979). 
El coeficiente de escurrimiento anual $K$ no debe ser confundido con el coeficiente de escurrimiento [de evento] $C$ utilizado en la hidrología urbana. Mientras que $K$ es una función del clima, $C$ es una función del grado de impermeabilidad de la cuenca en cuestión. La Figura 3 muestra un modelo conceptual de los coeficientes de escurrimiento, en el cual $K$ es una función de la relación entre la precipitación media anual y la precipitación anual global terrestre Pma/Pagt. El valor de esta última se estima en Pagt $=800 \mathrm{~mm}$ (Ponce, Pandey, \& Ercan, 2000).

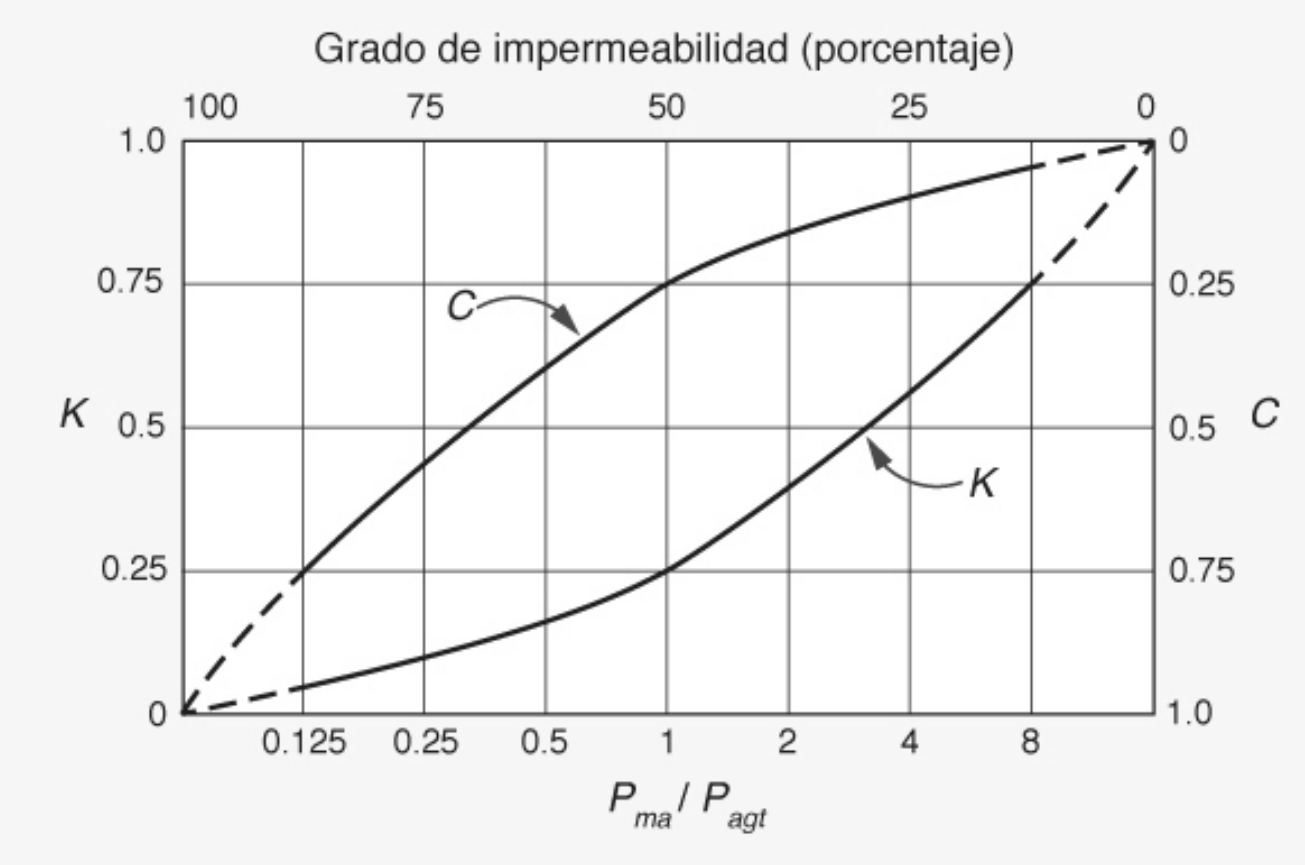

Figura 3. Modelo conceptual de coeficientes de escurrimiento.

La naturaleza ha diseñado el ciclo hidrológico de manera que la evaporación y el escurrimiento compartan la precipitación. En un extremo, para $K=0$, la escorrentía es nula y la evaporación es máxima; por lo tanto, no existen medios naturales para remover las sales. De esta forma, las sales se acumulan sin límite, circunscribiendo la diversidad de los ecosistemas. Tal es el caso de las cuencas endorreicas, normalmente situadas en el interior de los continentes. El resultado final es un desierto de sal, donde la vida es limitada, no sólo debido a la falta de agua, sino también por la acumulación continua de sal. Un ejemplo extremo de endorreísmo antropogénico es el del lago Salton, en California, donde en los últimos 80 años se ha formado un lago salado como resultado de la acumulación de drenaje agrícola en el Sumidero Salton, una depresión natural que yace debajo del nivel 
del mar. Las sales continúan acumulándose en el Lago Salton, sin que pueda vislumbrarse un fin aparente (Ponce, 2005).

En el otro extremo, en el caso de $K=1$, la evaporación es nula y el escurrimiento es máximo. Bajo este esquema, el sistema es claramente incapaz de propiciar y sostener cualquier tipo de vegetación. La vegetación requiere de un suministro constante de humedad, a fin de que las plantas vasculares puedan mantener su turgencia y evitar así el marchitamiento. El resultado es también un desierto, pues no hay posibilidad de que se establezca la vegetación.

Los casos mencionados son extremos, presentados aquí sólo para propósito de discusión. En la práctica, la naturaleza divide la precipitación en dos fracciones distintas: una para la evaporación, y otra para el escurrimiento. Las cantidades reales dependen no sólo del clima, sino también de la geología local y regional, la geomorfología, y de la posición relativa de la cuenca respecto a la masa continental.

Cuando el escurrimiento es mayor que el promedio, la vegetación tiende a cubrir toda la superficie del terreno; por lo tanto, la evaporación procede por dos trayectorias: (1) a través de la vegetación (evapotranspiración), y (2) directamente de los cuerpos de agua. En este caso, un rango típico de $K$ es de aproximadamente $0.4 \leq K \leq 0.6$. Un ejemplo de este caso es la cuenca de la selva amazónica (Figura 4), para la cual $K=0.52$, medido en el angostamiento de Obidos, en Pará, Brasil.

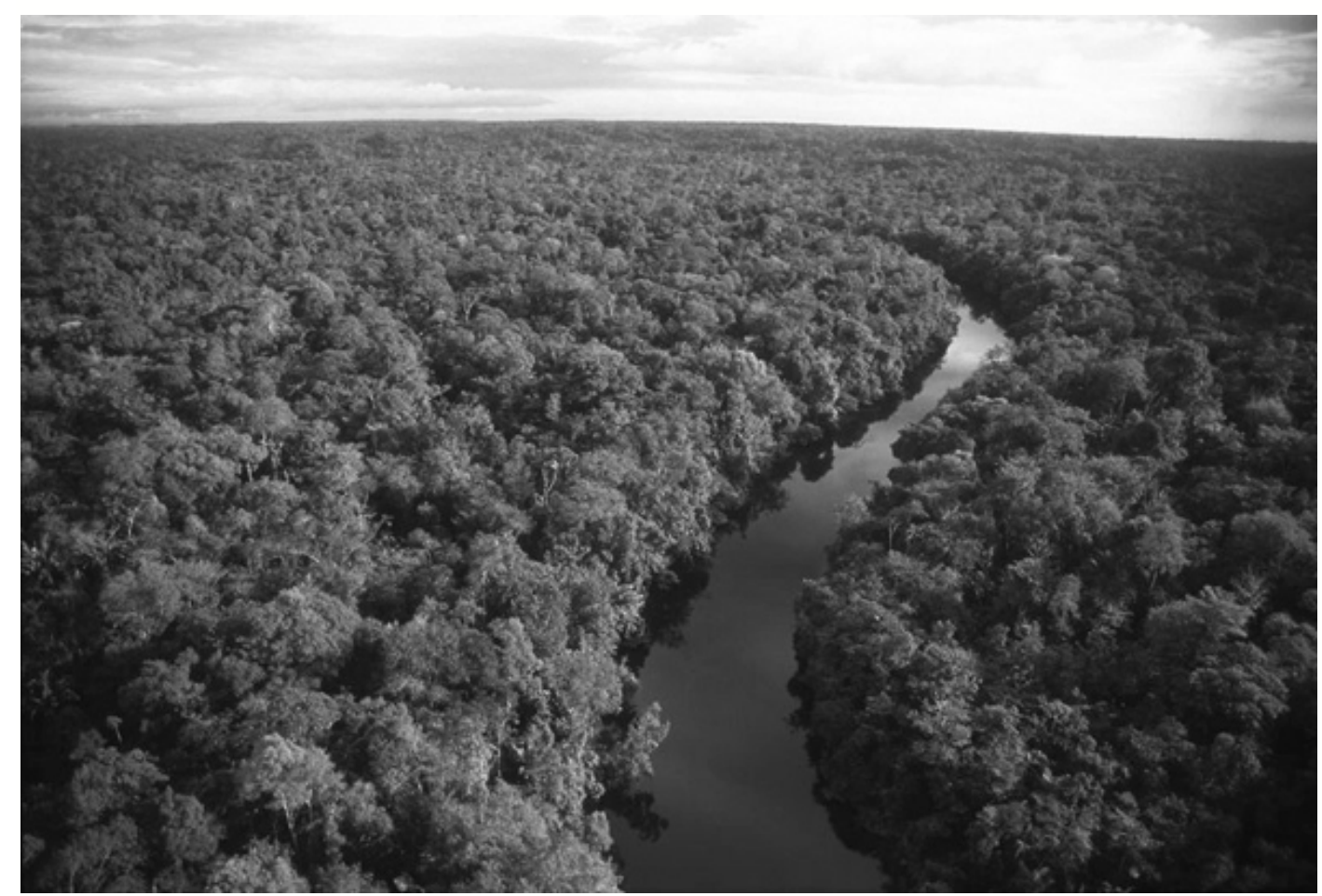


Figura 4. La selva amazónica (Wikimedia Commons, 2015).

Por el contrario, cuando el escurrimiento es menor que el promedio, la vegetación no cubre toda la superficie del terreno. En este caso, la evaporación procede a lo largo de tres trayectorias: (1) a través de la vegetación (evapotranspiración), (2) directamente de los cuerpos de agua, y (3) del suelo propiamente dicho (Figura 5). El rango típico de $K$ está en el rango $0.1 \leq K \leq 0.2$. Por ejemplo, en las inmediaciones de Ramona, en el centro del Condado de San Diego, California del Sur, Estados Unidos, $K=0.15$, confirmándose que este valor depende en alto grado de las condiciones climáticas locales y regionales.



Figura 5. Embalse Turner, en el condado de San Diego, California, Estados Unidos.

De acuerdo con el plan original de la naturaleza, los valores centrales de $K$ son más propicios para la salud del ecosistema $y$, por consiguiente, la diversidad ecológica. En un extremo, para $K \cong 0$, el sistema se caracteriza por la ausencia de escurrimiento y exceso de sales. En el otro extremo, para $K \cong 1$, el sistema muestra un exceso de escurrimiento y la ausencia de vegetación. En teoría, la distribución ideal debería corresponder a $K=0.3$, es decir, más o menos alrededor de un tercio para el escurrimiento, y los restantes dos tercios para la evaporación. En la práctica, esta condición corresponde aproximadamente al centro del espectro climático, el cual separa el clima semiárido (en el lado seco) del subhúmedo (en el lado húmedo) (Ponce et al., 2000). 
El comentario anterior sugiere que los coeficientes de escorrentía muy pequeños $(K \cong 0)$ son usualmente la excepción en los paisajes naturales con drenajes exorreicos. Una cuenca con un coeficiente de escurrimiento muy pequeño puede llevar a acumulaciones de sales, ya que el escaso escurrimiento es insuficiente para exportar todas las sales. Un ejemplo aclaratorio: las cuencas con asentamientos humanos importantes por lo general cuentan con coeficientes de escurrimiento en el rango $0.2 \leq K \leq 0.4$. Esto corresponde aproximadamente con climas semiáridos a subhúmedos, en los cuales la precipitación media anual varía entre $400 \mathrm{~mm}$ (16 pulgadas) y $1600 \mathrm{~mm}$ (64 pulgadas).

\section{El diseño del hombre}

En los últimos tiempos, pero en particular en los últimos cien años, los seres humanos han tendido a asentarse en regiones áridas y semiáridas, en las cuales el agua disponible suele ser insuficiente para satisfacer todas las necesidades, incluyendo las naturales y antrópicas. Las necesidades naturales son las del ecosistema predominante, el cual obviamente requiere de agua para su funcionamiento normal. Las necesidades antrópicas son las impuestas por los asentamientos humanos para la producción de alimentos, y usos domésticos e industriales. Por ejemplo, el límite entre las regiones semiáridas y áridas es de $400 \mathrm{~mm}$ (16 pulgadas) de precipitación media anual (Ponce et al., 2000), que debe corresponder más o menos (en una cuenca completamente exorreica) a un coeficiente de escorrentía $K=$ 0.2 . A menudo, esta cantidad de agua resulta insuficiente para satisfacer todas las necesidades previstas, como en el caso del sur de California, en Estados Unidos.

Un uso antrópico importante del agua es el consuntivo en la irrigación, para producir alimentos con el fin de satisfacer la creciente demanda. Los ecosistemas naturales son los primeros en tomar su parte de agua por evaporación y evapotranspiración; por lo tanto, esta cantidad ya está comprometida. Para los seres humanos, el único recurso parece ser la conversión del escurrimiento en evapotranspiración por medio de la irrigación. Esto resulta en una mayor cantidad de alimentos y otros productos, pero a costa de reducir el coeficiente de escurrimiento (obviamente, el uso consuntivo tiene que reducir la disponibilidad de agua en alguna otra parte del sistema). En efecto, la producción 
adicional de alimentos ha reducido la capacidad de la cuenca para remover y eliminar las sales.

Hay quienes sostienen que el agua de escurrimiento es demasiado valiosa para ser desperdiciada en los océanos. Bajo esta óptica equivocada, cada gota de precipitación convertida en escurrimiento debe ser almacenada y destinada a usos económicos. La irrigación es particularmente preocupante, pues si se desarrolla sin límite, puede conducir a la eventual desaparición del escurrimiento. Sobre todo en zonas áridas, cuando todo el escurrimiento se ha convertido en evapotranspiración, el coeficiente (de escurrimiento) se reduce a cero, esto lleva a la formación de un desierto infestado de sal (Figura 6).

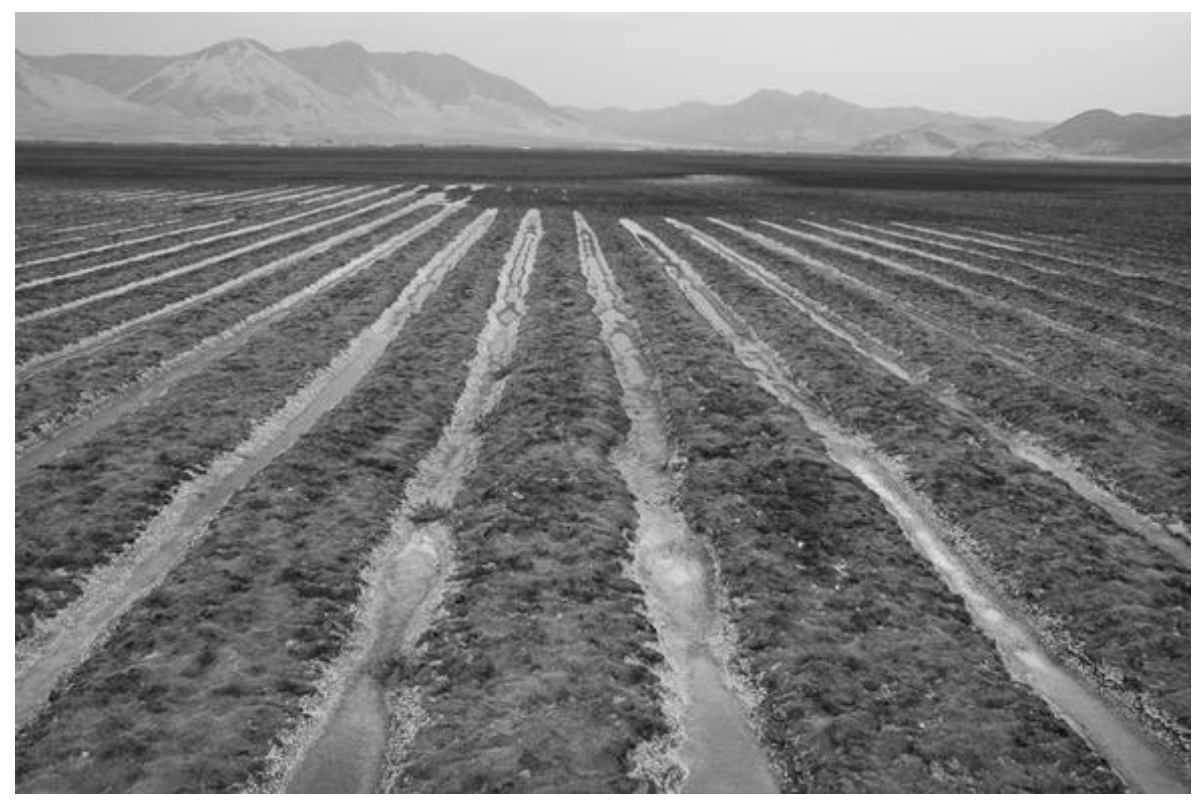

Figura 6. Salinización de un campo irrigado, valle de Chao, La Libertad, Perú.

La solución apunta a limitar, a través de una regulación apropiada, la conversión antrópica de escurrimiento en evapotranspiración. La irrigación debe limitarse a la cantidad que sea absolutamente necesaria. Cabe notar que, en regiones áridas y semiáridas, que es donde más se necesita la irrigación, la producción de alimentos resulta en menos escurrimiento, cuando en realidad se requiere de más escurrimiento para lograr la eliminación de todas las sales, incluyendo las sales adicionales producidas por la irrigación misma.

Una lección interesante a ser extraída de la historia es la de la poco conocida civilización hohokam, que floreció en el centro de Arizona, 
EUA, por más de 1200 años, hasta su inexplicable desaparición cerca del año 1450 d.C. (Figura 7) (Encyclopædia Britanica, 2015). Inundaciones, sequías y la acumulación extrema de sales se han usado en el intento de racionalizar la desaparición de los hohokam que, muy apropiadamente, en el idioma local pima significa "aquellos que han desaparecido." (Arizona Museum of Natural History, 2015). La salinización parece ser la causa más probable, pues las inundaciones enriquecen el suelo con nutrientes frescos y las sequías suelen ser temporales. Además, la salinización es un fenómeno generalizado y difícil de manejar, sobre todo en una región árida como el centro de Arizona.

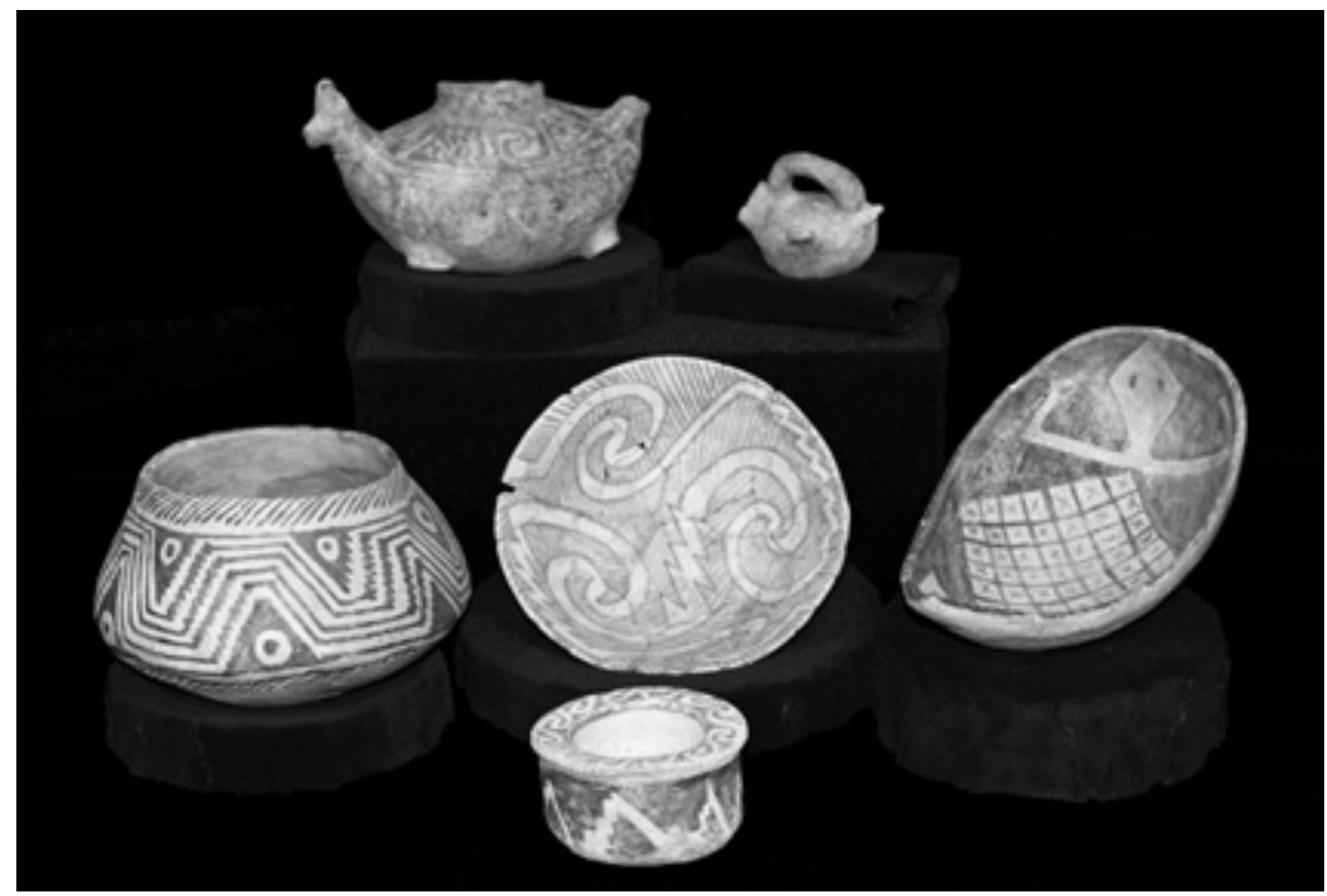

Figura 7. Cerámica hohokam (Arizona Museum of Natural History, 2015).

En las últimas dos décadas, la sostenibilidad de la irrigación ha sido examinada en detalle, en particular por el Manual de Práctica 71 de la Sociedad Americana de Ingenieros Civiles (American Society of Civil Engineers, 1990). Por un lado, hay quienes abogan por el retorno a la agricultura de secano, lo que implica el abandono de la irrigación como una empresa humana deseable. El problema comenzó con el asentamiento humano de las zonas áridas, en apariencia en búsqueda de más tierras productivas, además de un aire saludable y abundante sol. Descontando el abandono del asentamiento de zonas áridas, lo 
cual no es socialmente factible, el único camino que queda es la regulación de la irrigación. El objetivo debe ser reservar una porción del escurrimiento con el propósito específico de exportar las sales fuera de la cuenca (Ponce, 2014). Esto parece ser el único medio de asegurar la sostenibilidad.

\section{El balance de sales}

El concepto de equilibrio o balance de sales ayuda a explicar el conflicto entre el diseño de la naturaleza y el de los seres humanos. La naturaleza ha destinado las cuencas totalmente exorreicas para estar en equilibrio de sales, es decir, sin que experimenten una acumulación neta de sales en el tiempo. Por otro lado, las cuencas endorreicas no están en equilibrio, sino que están sometidas a un proceso continuo de acumulación de sales en el tiempo.

Es importante notar que el patrón de drenaje puede no estar siempre bien definido. En algunos casos, obedeciendo a la geomorfología local, las cuencas pueden ser semiendorreicas 0 semiexorreicas, dependiendo de cuál proceso es el predominante, la acumulación o la eliminación de sales, y en qué medida. Por ejemplo, la cuenca del río Alto Paraguay, la cual se encuentra ubicada cerca del centro geográfico de América del Sur, es, para todos los propósitos prácticos, una cuenca exorreica, con una descarga anual media de $1565 \mathrm{~m}^{3} / \mathrm{s}$ en su desembocadura, la confluencia con el río Apa, en Mato Groso del Sur, Brasil (Ponce, 1995). Sin embargo, la presencia demostrada de salinas, - lagunas de sal, en algunos lugares, muestra que no todas las áreas de la cuenca están efectivamente conectadas al sistema de drenaje superficial (Figuras 8 y 9) (Martins, 2012).

De hecho, la cuenca del río Alto Paraguay, mientras que parece estar en equilibrio de sales en el ámbito regional, en realidad puede no estarlo de forma local. Se concluye que en grandes cuencas subhúmedas con características de delta continental, como la del río Alto Paraguay, la geomorfología local juega un papel preponderante en el condicionamiento del endorreísmo. 


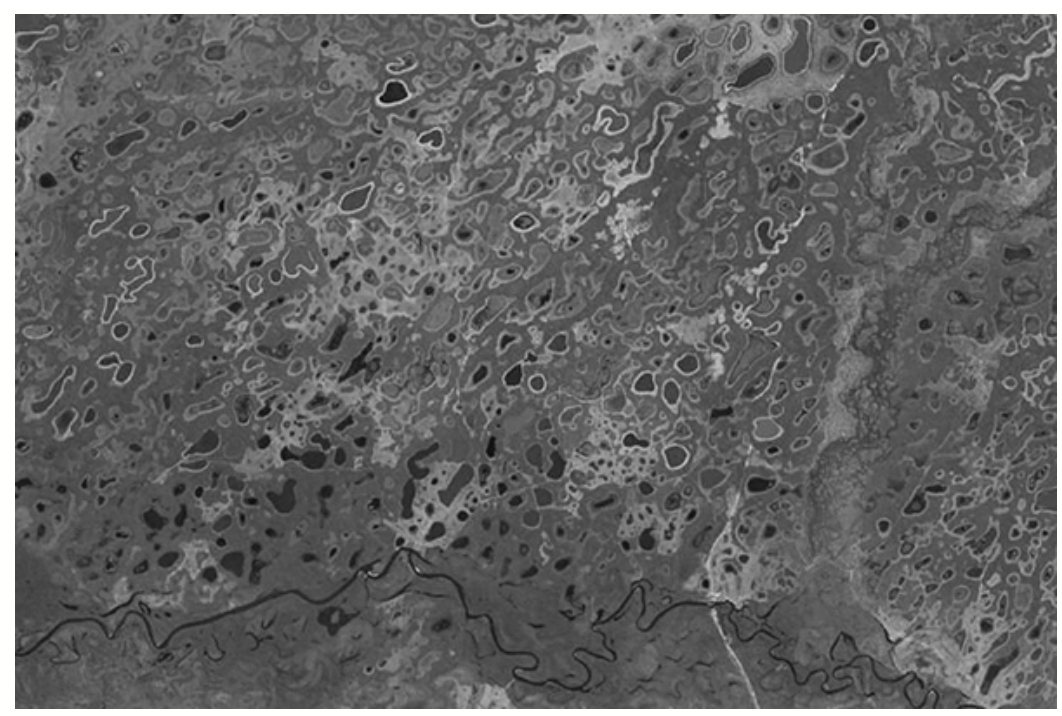

Figura 8. Vista aérea de las lagunas Salinas en la localidad de Río Negro, Mato Groso del Sur, Brasil (Google Earth, 2015).

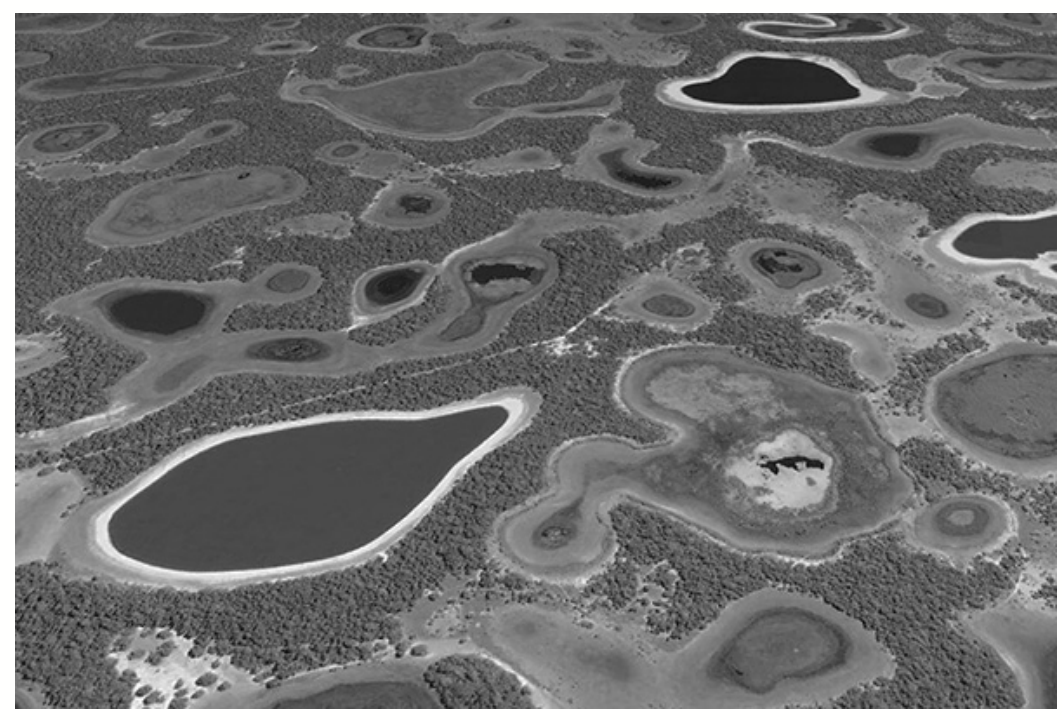

Figura 9. Detalle de las lagunas Salinas de la localidad de Río Negro, Mato Groso del Sur, Brasil (Google Earth, 2015).

La elección entre exorreismo y endorreísmo es clara. Las sales por lo general terminan degradando los ecosistemas naturales, en particular en las regiones áridas y semiáridas; por lo tanto, las cuencas exorreicas son preferibles sobre las endorreicas. Preservar o conservar el equilibrio de sales de una cuenca - presumiblemente lo más cercano a prístino como sea posible de manera realista- debe ser el objetivo principal del manejo sostenible de una cuenca. El ejemplo de la línea de salmuera Inland Empire, en la cuenca del río Santa Ana, en 
California del Sur, EUA, muestra que este objetivo es alcanzable (Santa Ana Watershed Project Authority, 2012). En este caso particular, la ubicación privilegiada de la cuenca, en la periferia continental, ha favorecido claramente el objetivo del balance de sales.

\section{La cuenca del lago Tulare}

Un ejemplo histórico aunque algo extremo del secuestro antrópico de escurrimiento y sales es el de la cuenca del lago Tulare, ubicada en el sur del Valle Central de California, EUA. Antes de 1850, es decir, previo al asentamiento y desarrollo del estado de California como tal, el lago Tulare era el más grande lago de agua dulce al oeste del río Mississippi, y el segundo más grande de EUA, basado en superficie. A la elevación de 216 pies $(66 \mathrm{~m})$, su nivel más alto de desbordamiento, registrado en 1862 y 1868 , el lago Tulare abarcaba 790 millas cuadradas (2 040 $\mathrm{km}^{2}$ ) (Figura 10) (ECORP Consulting, Inc., 2007). 




Figura 10. Cuenca del lago Tulare, Valle Central, California, EUA, 1874 (Wikipedia, 2015).

A través de la mayor parte del siglo XIX, la cuenca del lago Tulare funcionó como una cuenca semiendorreica, acumulando el escurrimiento local y regional de: (1) el Brazo Sur del río Kings; (2) el río Kaweah (Figura 11); (3) el río Tule, y (4) el río Kern. Durante periodos de inundación infrecuente, a altitudes superiores a 207 pies $(63 \mathrm{~m})$, el lago Tulare desbordaba en dirección norte, a través de Fresno Slough (humedal elongado Fresno), conectando con el río San Joaquín y eliminando así parte de sus sales. 




Figura 11. Río Kaweah, tributario del lago Tulare, California, EUA.

Durante el periodo 1850-1878 (29 años), el lago Tulare se desbordó 19 veces. El volumen total descargado durante ese lapso ha sido estimado en 1055000 acre-pies (ECORP Consulting, Inc., 2007). Después de 1878, debido a las desviaciones de los flujos tributarios para riego y usos municipales, no hubo más desbordamientos. En 1899, el lago quedó casi completamente seco, con la excepción de algunos humedales residuales e inundaciones ocasionales.

El desarrollo de la irrigación en la cuenca del lago Tulare ha causado que en los últimos 137 años todo el escurrimiento se haya retenido dentro de la cuenca y, con él, todas las sales. Además de las sales ya presentes en cantidades normales en el escurrimiento natural, el riego de tierras áridas crea nuevos residuos salinos (de calcio y sodio) mediante la desagregación de los suelos geológicamente jóvenes para extraer las sales útiles (magnesio y potasio) (Rhoades, Krueger, \& Reed, 1968). Por lo tanto, el desarrollo del riego representa un efecto negativo doble: menos escurrimiento disponible para transportar las sales regionales y más sal producida localmente.

En la cuenca del lago Tulare, las sales se han almacenado y continúan siendo almacenadas en sendas lagunas de evaporación (Figura 12). En los últimos años, con el fin de manejar las crecientes cantidades de sal, se han implementado varios hábitats artificiales para su uso por aves acuáticas (Figura 13). El efecto de esta operación masiva de colección y acumulación de sal en el medio ambiente circundante, tanto por encima como por debajo del terreno, aún no ha sido dilucidado. 


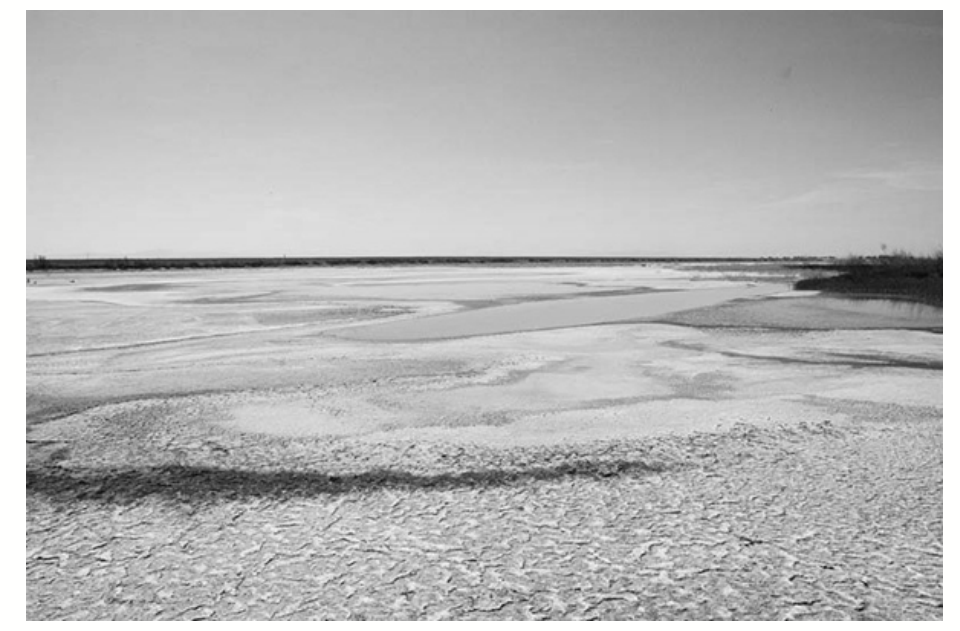

Figura 12. Laguna de evaporación sur, cuenca del lago Tulare, California, EUA.

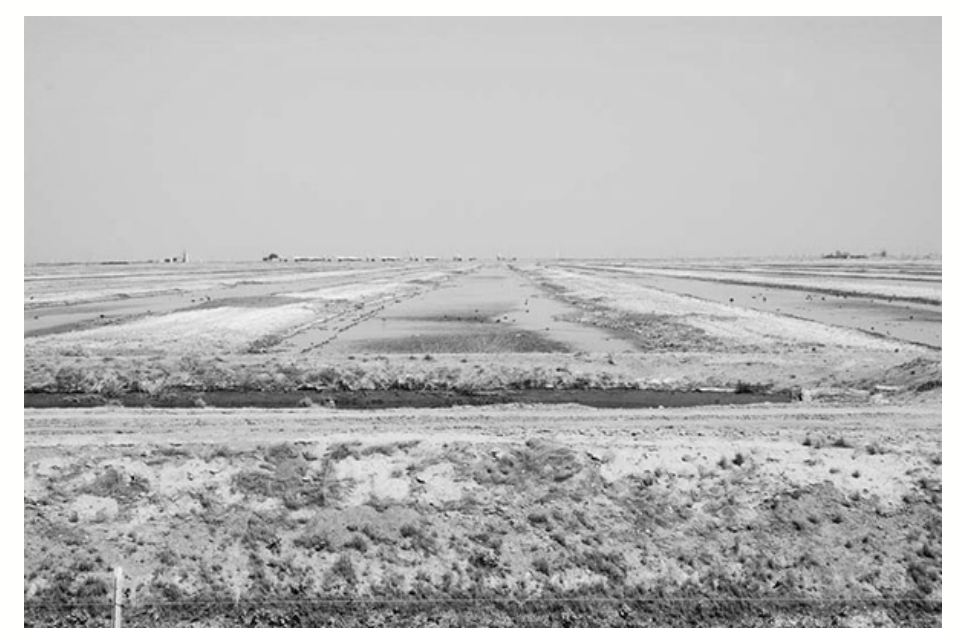

Figura 13. Hábitat para uso de aves acuáticas, cuenca del lago Tulare, California, EUA.

Pillsbury (1981) ha presentado un panorama bastante sombrío de las consecuencias eventuales de la acumulación de sales en lagunas de evaporación. Pillsbury afirma: "... Todos los depósitos de agua subterránea tienen una salida en algún lugar cerca de su extremo inferior. El agua salina en la cuenca de evaporación servirá para aumentar la carga hidráulica en las aguas salinas subyacentes, y por lo tanto, aumentar la descarga en la salida, causando estragos en las aguas superficiales y tierras ubicadas aguas abajo. Si la laguna de evaporación está encima de un terreno impermeable al agua dulce, 
este suelo se volverá gradualmente más permeable con las aguas salinas...".

Las ventajas y desventajas de los proyectos de irrigación, sobre todo en zonas áridas, están ahora claramente definidas. Si bien estos desarrollos resultan en una mayor cantidad de alimentos y otros productos agrícolas, dicho aumento es a costa de la generación de sales adicionales, lo cual demanda una eliminación adecuada. En ausencia de un plan de manejo apropiado, la acumulación de sales hará que el sistema se vuelva insostenible a mediano o largo plazos. Tal como lo describe Pillsbury, será cuestión de tiempo antes de que el sistema comience a mostrar sus fallas.

El caso del lago Tulare puede ser un ejemplo extremo de la retención antrópica de sales, pero de ninguna manera es un caso aislado. Todos los desarrollos de irrigación, en particular aquellos ubicados en zonas áridas, aumentan, ya sea: (a) la salinidad de las aguas superficiales ubicadas aguas abajo o (b) la salinidad de las aguas subterráneas locales (Pillsbury, 1981). Por lo tanto, las ventajas y desventajas de los proyectos de irrigación deben examinarse con cuidado antes del desarrollo. En muchos casos, cuando se tomen en cuenta todos los factores, la viabilidad de la irrigación puede resultar siendo elusiva (American Society of Civil Engineers, 1990).

\section{Conclusiones}

Este estudio conduce a las siguientes conclusiones:

Las rocas litosféricas son la fuente y origen de la mayoría de las sales del agua de mar. Las sales han sido transportadas a los océanos en el tiempo geológico por el flujo incesante de los ríos.

La biósfera terrestre retiene la mayor parte del potasio y cantidades apreciables de magnesio. Calcio y sodio parecen ser poco necesitados por la biósfera terrestre.

Por cada tonelada de roca intemperizada y erosionada, se liberan a la hidrósfera unos $55 \mathrm{~kg}$ de cationes de sal. 
El océano es un sumidero de calcio, el cual es empleado por una variedad de organismos marinos para la construcción de conchas y esqueletos.

El único catión elemental de sal que permanece en gran parte sin usarse, inclusive en los océanos, es el sodio. No existe un proceso biológico conocido que elimine el sodio disuelto en los océanos.

Los ríos son los agentes mediante los cuales la naturaleza dispone de sus residuos de sal. En efecto, los ríos son los exportadores naturales de sal a los océanos (Pillsbury, 1981).

Al reducir el caudal de un río a través de la irrigación, el efecto neto es la conversión gradual de un drenaje exorreico en uno endorreico, afectando de forma negativa el balance natural de sales.

Los valores centrales del coeficiente de escurrimiento son más propicios para fomentar la salud de los ecosistemas y la diversidad ecológica. El valor ideal corresponde a $K=0.3$, es decir, alrededor de un tercio de la precipitación convertida en escurrimiento, y los dos tercios restantes en evaporación y evapotranspiración.

Cuando es desarrollada sin ningún límite, la irrigación conduce a la eventual desaparición de todo el escurrimiento. En este caso, el coeficiente de escurrimiento anual se reduce a cero, llevando a la formación de un desierto infestado de sales.

Todo desarrollo de irrigación aumenta, ya sea: (a) la salinidad de las aguas superficiales ubicadas aguas abajo o (b) la salinidad de las aguas subterráneas locales (Pillsbury, 1981). Por lo tanto, las ventajas y desventajas de los proyectos de irrigación deben ser examinadas con cuidado antes del desarrollo.

En resumen, iones de sodio y calcio son producidos por la desintegración de la litósfera en cantidades muy por encima de las que podrían ser asimiladas por la biósfera terrestre. En drenajes exorreicos, el residuo resultante ha sido (y está siendo) transportado al océano por los ríos. En drenajes endorreicos, los residuos salinos se han acumulado en el interior de los continentes, formando cuencas cerradas infestadas de sal. En general, el exorreismo es preferible al endorreísmo, porque lleva a ecosistemas más saludables, con una mayor diversidad biológica.

El desarrollo de irrigación convierte la escorrentía en evaporación, reduciendo así la cantidad de agua disponible para el lavado de las sales. Además, sobre todo en regiones áridas y semiáridas, que es donde más se necesita, la irrigación moviliza nuevas sales a través de la biodegradación adicional de suelos geológicamente jóvenes 
(Rhoades et al., 1968). En el límite, cuando todo el escurrimiento es secuestrado y convertido en evaporación, no queda ningún escurrimiento disponible para llevar las sales al océano. En cuanto el sistema se conduce de forma gradual hacia este límite, se vuelve insostenible, no por falta de agua para apoyar la vegetación, sino por falta de agua para eliminar las sales.

Prestando atención a los principios de la hidrología, aquí postulamos que la naturaleza tenía sin duda un plan diferente: en promedio, dos tercios del agua disponible para el ecosistema, y el tercio restante para el lavado de todas las sales, incluyendo sales regionales y locales (Pillsbury, 1981). La estrategia equivocada de retener todo o la mayor parte del escurrimiento, con el argumento de que constituye un recurso muy valioso, sólo puede conducir a la eventual conversión de cuencas continentales periféricas en cuencas artificialmente infestadas de sal. Cabe afirmar que éste no era el diseño original de la naturaleza.

\section{Referencias}

American Society of Civil Engineers. (1990). Irrigated agriculture: Is it sustainable? Chapter 28. In: Agricultural Salinity Assessment and Management. (Manuals and Reports on Engineering Practice No. 71). New York: American Society of Civil Engineers.

Arizona Museum of Natural History. (2015). The Hohokam. Recuperado de http://arizonamuseumofnaturalhistory.org//arch/hohokam.aspx

Deevey, E. S. (Sept. 1970). Mineral cycles. Scientific American, 223(3), 149-158.

ECORP Consulting, Inc. (2007). Tulare Lake basin hydrology and hydrography: A summary of the movement of water and aquatic species. Washington, DC, USA: U.S. Environmental Protection Agency.

Encyclopædia Britannica. (2015). Hohokam culture. Recuperado de http://www.britannica.com/topic/Hohokam-culture

Google Earth. (2015). Imágenes. Consultado el 19 de febrero del 2015.

L'vovich, M. I. (1979). World water resources and their future. Traducción del idioma ruso por Raymond L. Nace. Washington, DC, USA: American Geophysical Union.

Martins, E. R. C. (2012). Tipologías de lagoas salinas no Pantanal de Nhecolândia (MS) (Disertación doctoral). Universidade de São Paulo, São Paulo, Brasil. 
Penman, H. L. (Sept. 1970). The water cycle. Scientific American, 223(3), 99-108.

Pillsbury, A. F. (July, 1981). The salinity of rivers. Scientific American, 245(1), 54-65.

Ponce, V. M. (1995). Hydrologic and environmental impact of the Parana-Paraguay Waterway on the Pantanal of Mato Grosso, Brazil: A reference study. San Diego, USA: San Diego State University.

Ponce, V. M., Pandey, R. P., \& Ercan, S. (April, 2000). Characterization of drought across climatic spectrum. Journal of Hydrologic Engineering, ASCE, 5(2), 222-224.

Ponce, V. M. (2005). The Salton Sea: An assessment. Recuperado de http://ponce.sdsu.edu/salton_sea_assessment.html

Ponce, V. M. (2009). Sustainable runoff for basin salt balance. Recuperado de http://ponce.sdsu.edu/sustainable_runoff_for_basin_salt_balance.ht $\mathrm{ml}$

Ponce, V. M. (2014). Ecohydroclimatological research: The case for geomorphology. Recuperado de http://ponce.sdsu.edu/geomorphology.html\#geomorphology

Rhoades, J. D., Krueger, D. B., \& Reed, M. J. (1968). The effect of soilmineral weathering on the sodium hazard of irrigation waters. Soil Science Society of America Proceedings, 32, 643-647.

Santa Ana Watershed Project Authority. (2012). Inland Empire Brine Line. Recuperado de http://www.sawpa.org/brineline

Swenson, H. (2015). Why is the ocean salty? U.S. Geological Survey. Recuperado de http://ponce.sdsu.edu/usgs_why_is_the_ocean_salty/usgs_why_is_t he_ocean_salty.html

Trotamexico.com. (2015). Guerrero Negro, la salina más grande del mundo. Recuperado de http://www.trotamexico.com/es/articulo/bajacalifornia-sur/guerrero-negro/informacion-general/lu/guerrero-negrola-salina-mas-grande-del-mundo/

Wikipedia. (2015). Tulare Lake. Recuperado de https://en.wikipedia.org/wiki/Tulare_Lake

Wikimedia Commons. (2015). Selva amazónica. Recuperado de https://commons.wikimedia.org/wiki/Main_Page 